\title{
Publisher Correction: K33-linked polyubiquitination of Zap70 by Nrdp1 controls CD8+ Tcell activation
}

Mingjin Yang, Taoyong Chen, Xuelian Li, Zhou Yu, Songqing Tang, Chen Wang, Yan Gu, Yanfang Liu, Sheng Xu, Weihua Li, Xuemin Zhang, Jianli Wang and Xuetao Cao

Correction to: Nature Immunology https://doi.org/10.1038/ni.3258, published online 21 September 2015.

In the version of this article initially published, Fig. 2a, lower right panel, duplicated the flow cytometric profile in Fig. $2 \mathrm{~b}$, upper left panel. The correct cytometric profile is shown below. The error has not been corrected in the original article.
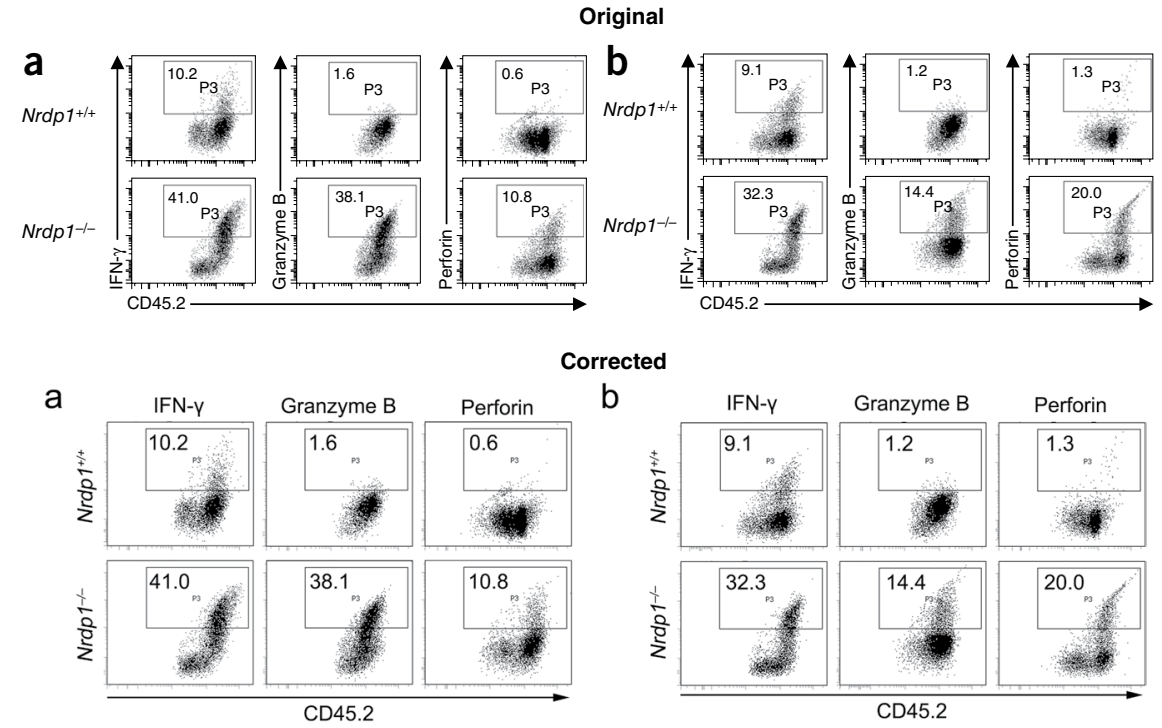

b

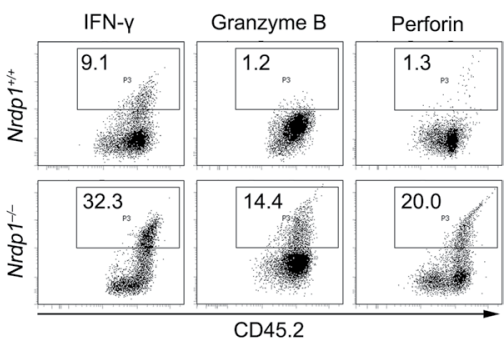

Fig. 2 | Original and Corrected.

Published online: 7 February 2020

https://doi.org/10.1038/s41590-020-0600-1

(c) The Author(s), under exclusive licence to Springer Nature America, Inc. 2020 